\title{
Changes in articular cartilage after meniscectomy and meniscus replacement using a biodegradable porous polymer implant
}

\author{
Gerjon Hannink - Tony G. van Tienen • \\ Arend Jan Schouten • Pieter Buma
}

Received: 9 February 2010/Accepted: 2 August 2010/Published online: 28 August 2010

(c) The Author(s) 2010. This article is published with open access at Springerlink.com

\begin{abstract}
Purpose To evaluate the long-term effects of implantation of a biodegradable polymer meniscus implant on articular cartilage degeneration and compare this to articular cartilage degeneration after meniscectomy.

Methods Porous polymer polycaprolacton-based polyurethane meniscus implants were implanted for 6 or 24 months in the lateral compartment of Beagle dog knees. Contralateral knees were meniscectomized, or left intact and served as controls. Articular cartilage degeneration was evaluated in detail using India ink staining, routine histology, immunochemistry for denatured $(\mathrm{Col} 2-3 / 4 \mathrm{M})$ and cleaved (Col2-3/4 $\left.\mathrm{C}_{\text {short }}\right)$ type II collagen, Mankin's grading system, and cartilage thickness measurements.

Results Histologically, fibrillation and substantial immunohistochemical staining for both denatured and cleaved type II collagen were found in all three treatment groups. The cartilage of the three groups showed identical degradation patterns. In the 24 months implant group, degradation appeared to be more severe when compared to the 6 months implant group and meniscectomy group.
\end{abstract}

G. Hannink $(\bowtie) \cdot$ P. Buma

Orthopaedic Research Lab, Department of Orthopaedics,

Radboud University Nijmegen Medical Centre,

P.O. Box 9101, 6500 HB Nijmegen, The Netherlands

e-mail: g.hannink@orthop.umcn.nl

T. G. van Tienen

Sint Maartenskliniek, P.O. Box 9011,

6500 GM Nijmegen, The Netherlands

\section{A. J. Schouten}

Polymer Chemistry, Faculty of Mathematics and

Natural Sciences, University of Groningen, Nijenborgh 4,

9747 AG Groningen, The Netherlands
Significantly more cartilage damage (India ink staining, Mankin's grading system, and cartilage thickness measurements) was found in the 24 months implant group compared to the 6 months implant group and meniscectomy group.

Conclusion Degradation of the cartilage matrix was the result of both mechanical overloading as well as localized cell-mediated degradation. The degeneration patterns were highly variable between animals. Clinical application of a porous polymer implant for total meniscus replacement is not supported by this study.

Keywords Porous polymer implant - Meniscectomy . Meniscus replacement - Cartilage degeneration · Cartilage thickness · India ink

\section{Introduction}

The meniscus plays a critical role in load transmission, stability and energy dissipation in the knee joint. Loss of the meniscus leads to joint degeneration and osteoarthritis. An increased understanding of the degenerative changes that occur after meniscectomy made clear that it is beneficial to save as much meniscal tissue as possible [20]. Meniscal repair has become a standard procedure, and partial resection of damaged menisci should be performed as sparingly as possible. However, not all damaged menisci can be treated by partial resection or by repair, making a total meniscectomy inevitable. In these cases, replacement of the resected meniscal tissue by an implant might avoid the articular cartilage degeneration [36]. Therefore, the long-term success of meniscus replacement depends on the prevention of degenerative osteoarthritic changes within the articular cartilage of the knee. 
Different types of meniscal substitutes, such as allografts [27, 31, 35, 43], fat tissue [15], tendons [16], perichondral tissue [3, 4], biodegradable scaffolds [10, 17, 38, $39,45]$, and permanent synthetic scaffolds [14, 22, 23, 34], have been used in experimental and clinical studies. Although encouraging, much of the outcome data following meniscal replacement have been subjective and of limited follow-up.

With regard to the graft material, to date, fresh frozen and cryopreserved allografts seem to provide the best a priori conditions for the prevention of degenerative arthritis $[5,12,25,30]$. However, problems related to the availability, preservation techniques, the possible transfer of diseases, the individual shaping of the implant, and possible immunologic reactions to the implant are recognized worldwide [30].

To avoid all problems related to the above-mentioned replacement techniques, our long-term aim is to generate a completely new meniscus by in vivo tissue engineering. Therefore, we developed a porous polycaprolacton-based polyurethane (PCLPU) implant with nontoxic degradation products [8]. This implant acts as a temporarily scaffold to enable the regeneration of new meniscus tissue in time by slow degradation of the polymer and simultaneous differentiation of the ingrown fibro-vascular tissue into the typical avascular meniscus fibro-cartilage.

Cartilage degeneration has been investigated in relatively few studies compared to the total amount of literature concerning meniscal replacements $[1,7,24,28,32$, 37]. In a previous study, we compared articular cartilage damage after meniscectomy and after implantation of a porous polymer, both after 3 and 6 months [38]. No significant differences in cartilage degeneration could be observed between the meniscectomy and the implant groups, and between the follow-up periods. In other words, the porous polymer implant could not prevent cartilage degeneration. It was speculated that the cartilage degeneration merely had taken place during the first months after implantation. At that time, the relatively rough prosthetic surface, not yet covered with tissue, was in direct contact with the articular cartilage. However, between 3 and 6 months after implantation, the whole implant was covered with a tissue layer. In the long-term, when the implant is completely infiltrated and surrounded with tissue, the gliding characteristics of the construct might improve. Consequently, the progression of the cartilage degeneration might end.

Therefore, the aim of the present study was to evaluate the long-term effects of implantation of a polymer implant compared to those of meniscectomy on articular cartilage degeneration. The long-term tissue differentiation, changes in implant stiffness, and foreign body reactions have been published earlier [41].

\section{Materials and methods}

Implant

Porous PCLPU meniscus implants were produced using a Teflon mold (Teflon PTFE, DuPont, Wilmington, DE, USA). The implants were provided with an interstice for the popliteus tendon (Fig. 1). The PCLPU consists of a hard segment of 1,4-butanediisocyanate and butanediol and a soft segment of poly ( $\varepsilon$-caprolactone) $[8,9]$. The pores were created by mixing the polymer solvent solution with salt crystals ranging in size from 150 to 355 microns, which were washed away after complete polymerization of the scaffold. The macropores were directly interconnected to achieve a high permeability of the polymer. The porosity was $81.4 \%$, and the compression modulus at $20 \%$ compression was $300 \mathrm{kPa}[8]$.

\section{Experimental design}

Four experimental groups were defined: (1) native meniscus group, (2) 6 months polymer implant group, (3) 24 months polymer implant group, and (4) 24 months meniscectomy group (Fig. 2). Thirteen adult male and female Beagle dogs (mean weight $12.5 \pm 0.2 \mathrm{~kg}$ ) were bilaterally operated, however not in one session. In the first session, six dogs underwent a lateral meniscectomy. Seven dogs underwent the implantation of a polymer meniscus immediately after lateral meniscectomy. One of these dogs underwent a lateral meniscectomy in the contralateral knee. The treatments, polymer implant or meniscectomy for 24 months, were randomly assigned to either the right or left knee. In the second session, six dogs underwent an implantation of a polymer meniscus immediately after lateral meniscectomy. The remaining six knees were left intact and served as control. The treatments, polymer implant for 6 months or control, were randomly assigned to the dogs. The Animal Experimentation Committee of the Radboud University Nijmegen approved all procedures.

\section{Surgical procedure}

Preoperatively, all animals received antibiotics $(12.5 \mathrm{mg} / \mathrm{kg})$ (amoxillin, Centrafarm BV, Etten-Leur, The Netherlands) and analgetics $(5 \mu \mathrm{g} / \mathrm{kg})$ (Fentanyl Bipharma, Hameln Pharmaceuticals $\mathrm{GmbH}$, Hameln, Germany). All animals were anesthetized by intravenous administration of $4 \mathrm{mg} / \mathrm{kg}$ propofol (B. Braun Melsungen AG, Melsungen, Germany) and maintained after intubation with nitrous oxide, oxygen, and isoflurane $(1.5 \%)$.

Under aseptic conditions, the lateral meniscus was approached by a lateral incision of the knee joint capsule without detachment of any ligament. Using a Beaver 


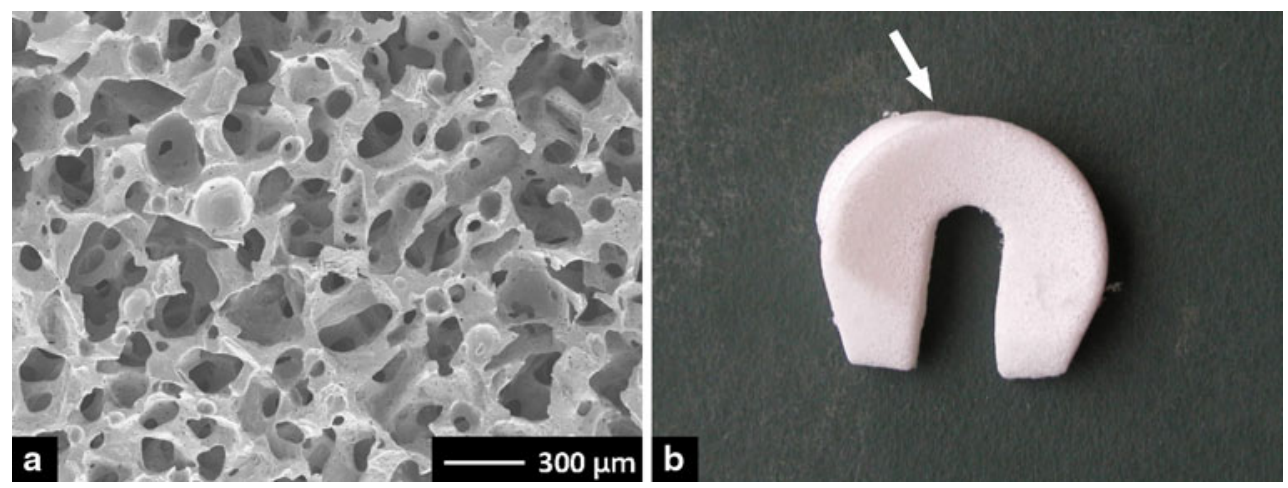

Fig. 1 a Scanning electron microscopy image of a cross section of the polycaprolacton-based polyurethane (PCLPU) implant. b Porous PCLPU meniscus implant provided with an interstice for the popliteus tendon (arrow)

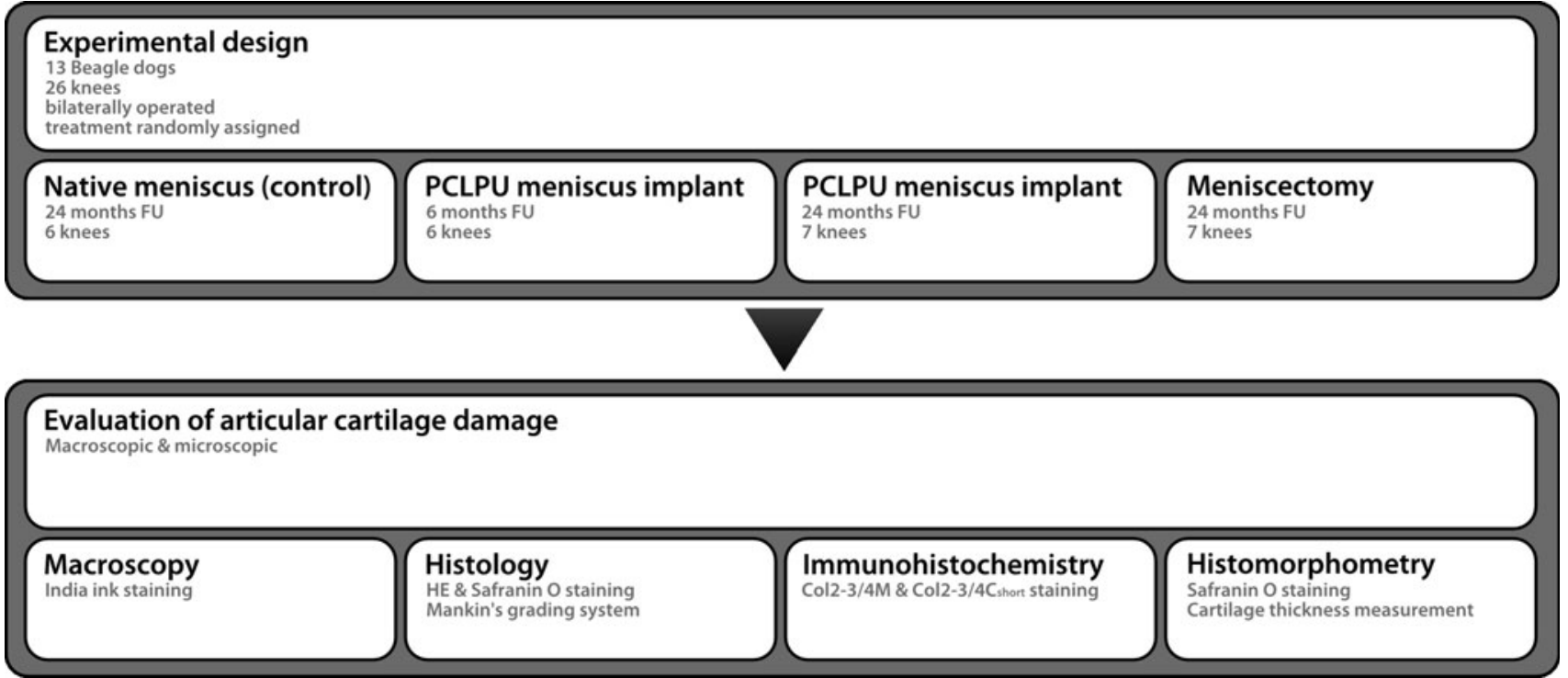

Fig. 2 Schematic overview of experimental design

eyeblade (Waltham, MA, USA), the meniscus was separated from its anterior and posterior attachments. Two drill holes were created originating from the lateral tibial side to the former attachment of the anterior and posterior horns of the native meniscus. Two bonded nondegradable sutures were led through the two horns of the implant. The sutures were pulled through the drill holes in the tibia. The periphery of the implant was sutured to the peripheral knee joint capsule using resorbable bonded sutures (Vicryl 2/0 Ethicon, Johnson \& Johnson Medical BV, Amersfoort, The Netherlands) to achieve close contact between implant and synovial tissues. Afterward, the capsule and skin were closed. After the implantation procedure, the animals received subcutaneous injections of antibiotics $(15 \mathrm{mg} / \mathrm{kg})$ (Excenel, Pharmacia \& Upjohn Animal Health BV, Woerden, The Netherlands) and analgetics $(1.1 \mathrm{mg} / \mathrm{kg})$ (Fynadine, Schering Plough Animal Health Benelux, Utrecht, The Netherlands) two times. The dogs were not immobilized and were allowed to walk as soon as possible. At the set time points, the dogs were killed with an overdose of sodium pentobarbital (Nembutal, CEVA Sante Animale, Maasluis, The Netherlands) (1 mL/kg).

Evaluation of articular cartilage

After radiographs were taken, all knee joints were disarticulated, and digital photographs were taken using standardized lighting conditions. Menisci, tissues that had been formed in the meniscectomy group, or the polymer implants were carefully removed. India ink (Rotring International $\mathrm{GmbH} \&$ Co., Hamburg, Germany) was applied to the articular surfaces for $30 \mathrm{~s}$, then washed off using running tap water for $60 \mathrm{~s}$ to identify areas of cartilage fibrillation. Areas of India ink uptake were photographed to enable visualization and analysis of the fibrillated areas on tibia and femur [2]. The ink-stained area 
was measured using an interactive, computer-controlled, image analysis system (analySIS Soft Imaging System, GmbH, Münster, Germany).

\section{Histology}

The tibial plateaus and femoral condyles were dissected in equal proportions (Fig. 3). The tibial plateaus were divided into a lateral and a medial part, which were subsequently divided into an anterior and posterior half. The anterior half was processed for routine histology and the posterior half for immunohistochemistry. The femur condyles were also divided in a medial half for immunohistochemistry and a lateral half for routine histology. Sections of the tibia were cut in mid-transverse plane and sections of the femur in mid-sagittal plane.

The anterior halves of the tibial plateaus and the lateral halves of the femoral condyles were fixed in buffered $4 \%$ formaldehyde ( $\mathrm{pH}$ 7.4) and decalcified in 10\% EDTA (Titriplex III, Merck, Darmstadt, Germany). After extensive rinsing with tap water, tissue blocks were dehydrated in alcohol and embedded in PMMA for 2 days at $37^{\circ} \mathrm{C}$. Specimens were cut using a microtome (Leica RM 2155 , Leica Microsystems Nederland BV, Rijswijk, The Netherlands) into 7 - $\mu$ m-thick sections. The sections were stained with hematoxylin-eosin (HE) and safranin O. Degenerative changes were scored according to Mankin's grading system [19].

\section{Immunohistochemistry}

To determine the location of damaged collagen, the samples were stained using $\mathrm{Col} 2-3 / 4 \mathrm{M}$ and $\mathrm{Col} 2-3 / 4 \mathrm{C}_{\text {short }}$ antibodies. Col $2-3 / 4 \mathrm{M}$ is a nonspecific indicator of collagen molecule damage, tagging an epitope within the triplehelix of the collagen molecule after the molecule is denatured. Col $2-3 / 4 \mathrm{C}_{\text {short }}$ is a rabbit polyclonal antibody directed against the $\mathrm{COOH}$ terminus of the three-quarter fragment, which is generated very specifically by the cleavage of native type II collagen by mammalian collagenases [42].
Using these antibodies, distinction can be made between mechanical damage $(\mathrm{Col} 2-3 / 4 \mathrm{M})$ and enzymatic cleavage $\left(\mathrm{Col} 2-3 / 4 \mathrm{C}_{\text {short }}\right)$. The staining procedures have been described in detail elsewhere [42]. Adjacent sections were stained with safranin $\mathrm{O}$.

\section{Cartilage thickness}

The thickness measurements were made according to a previously described method [26]. The cartilage thickness was measured in the central zone of the tibial plateau and in the corresponding zone on the femoral condyle. For each specimen, five equally distanced $(100 \mu \mathrm{m})$, safranin O-stained sections were measured at a $12.5 \times$ magnification using custom-made software. Manually, a polyline roughly delimiting the cartilage from the subchondral bone was produced. This polyline was converted to a b-spline consisting of a fixed number of points (500 points/section). Two parallel polylines were constructed from this b-spline. One of these polylines was offset to the inside, the other to the outside. A series of straight line segments was generated between each pair of points on these polylines. On each line segment, two points were automatically calculated (Fig. 4). The first one represents the transition between calcified cartilage and cartilage (tidemark level). The second one represents the outside edge of the cartilage. If necessary, these points could be corrected manually. The distance between these points was considered as the cartilage thickness along the line segment. The cartilage thickness was calculated as the mean length of all the segments generated. All sections were investigated blinded and in random order.

\section{Statistical analysis}

The ink-stained surface area, Mankin score, and cartilage thickness datasets were evaluated nonparametrically as normal distribution could not be assumed for all parameters. Between-treatment group datasets were evaluated with Kruskal-Wallis ANOVA by ranks followed by Wilcoxon signed-rank test. Within-treatment group datasets were
Fig. 3 Schematic drawing showing the dissection of tibial plateaus and femoral condyles for histological and immunohistochemical analysis
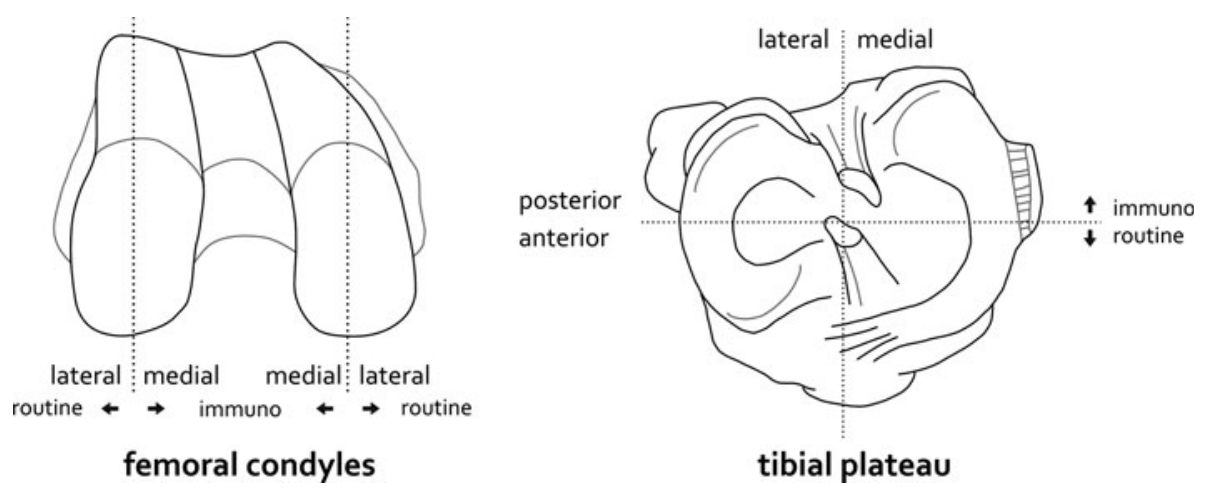
Fig. 4 Schematic representation of cartilage thickness measurements. A series of straight line segments perpendicular to both of the polylines were generated. On each line segment, two points were automatically calculated. One point represents the transition between calcified cartilage and cartilage (tidemark), and the other point represents the outer edge of the cartilage. The distance between these points was considered as the cartilage thickness along the line segment. The mean length of all line segments between the two points was calculated as overall cartilage thickness

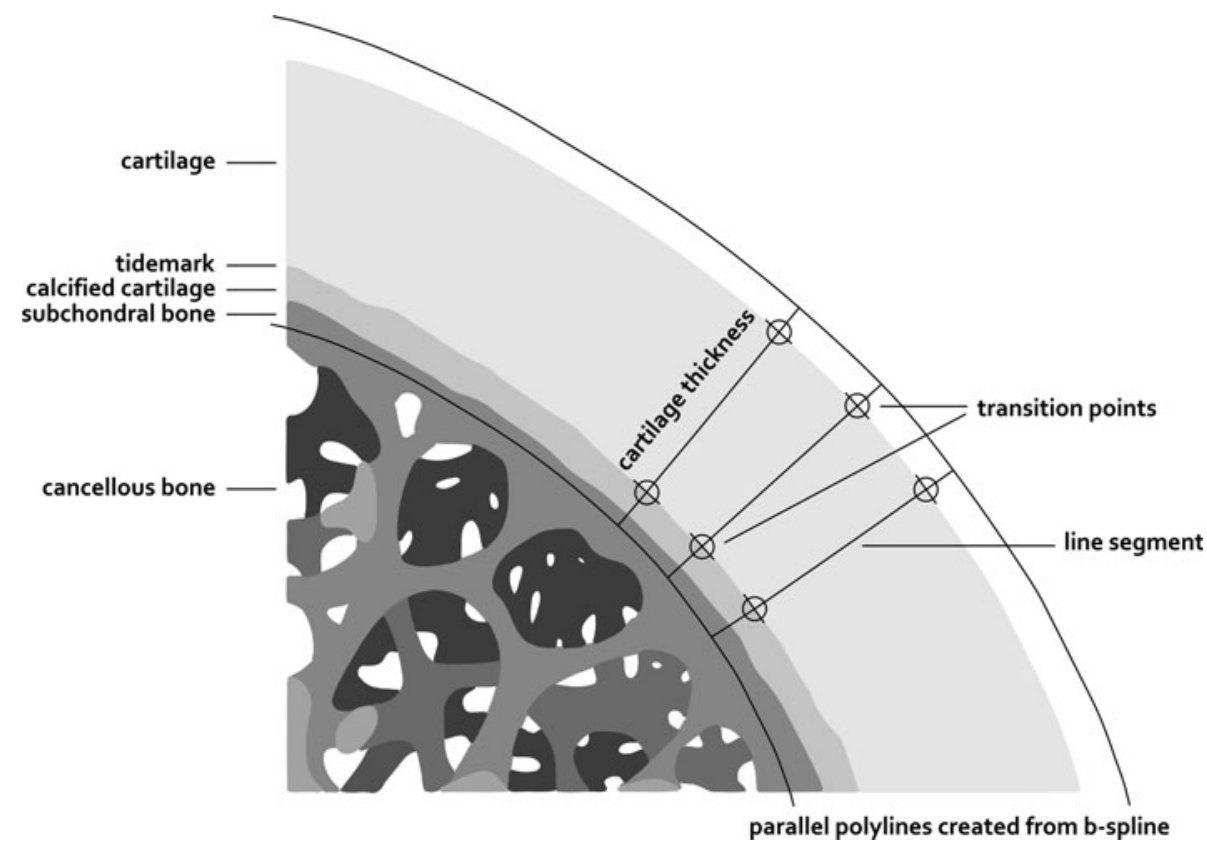

evaluated using Wilcoxon rank-sum test. For all datasets, differences were considered statistically significant for $P$ values $<0.05$. Statistical analysis was performed using SPSS 16.0 software (SPSS Inc., Chicago, IL, USA).

\section{Results}

\section{Macroscopic observations}

Postoperatively, all dogs established a normal gait pattern within 2 weeks. Two dogs had a delay of 3-4 days. One dog was successfully treated for a suspected infection of the knee joint. While harvesting the menisci, it was noticed that the implants were severely damaged in all knees. The implants appeared to be in near anatomic position and well attached to the peripheral synovial tissue with minimal synovial overgrowth. In the meniscectomy group, a fibrous structure was formed at the original location of the meniscus. All tissues were available for evaluation.

The India ink staining allowed clear demarcation of subtle cartilage defects as well as the zone of injury (Fig. 5). On both femur and tibia, ink-stained surface areas were significantly smaller on the nonoperated, medial side compared to the ink-stained areas on the lateral side, except for the native meniscus group (Table 1).

\section{Histology}

In the nonoperated medial compartments and in the native meniscus group, safranin $\mathrm{O}$ staining was almost up to the surface of the cartilage. Only in the upper thin superficial

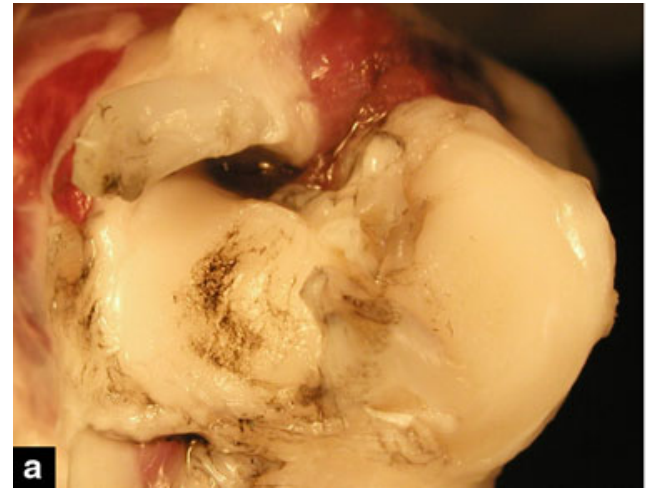

Fig. 5 India ink-stained tibial plateau a and femoral condyles $\mathbf{b}$ from a 24 months implant group. In the central weight-bearing zone of the lateral tibial plateau and corresponding area on lateral femoral

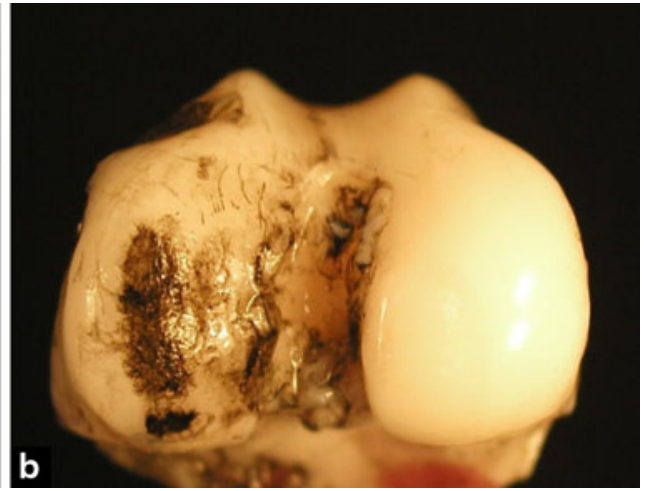

condyle, articular cartilage damage can be identified as the area of ink uptake. Medial tibial plateau and femoral condyle show no areas of ink uptake 
Table 1 India ink-stained surface area $\left(\mathrm{mm}^{2}\right)$

\begin{tabular}{|c|c|c|c|c|c|c|}
\hline \multirow[t]{2}{*}{ Treatment } & \multicolumn{3}{|l|}{ Femur } & \multicolumn{3}{|l|}{ Tibia } \\
\hline & Medial & Lateral & $P$ value & Medial & Lateral & $P$ value \\
\hline Native meniscus & $0.0(0.0-0.0)$ & $0.0(0.0-0.0)^{*}$ & 1.0 & $4.7(0.0-10.7)^{* *}$ & $6.2(0.0-9.8)^{\dagger, \ddagger, \#}$ & 0.9 \\
\hline 6 months & $0.0(0.0-0.0)$ & $20.9(11.8-32.5)$ & 0.001 & $6.9(1.9-8.2)$ & $21.5(17.7-26.4)^{\dagger}$ & 0.001 \\
\hline 24 months & $0.0(0.0-1.1)$ & $51.4(19.3-53.9)^{*}$ & 0.001 & $8.0(3.5-14.8)^{* *}$ & $27.0(19.1-54.2)^{*}$ & 0.004 \\
\hline Meniscectomy & $0.0(0.0-0.0)$ & $29.5(0-69.7)$ & 0.02 & $7.0(2.8-10.1)$ & $30.7(32.0-40.0)^{\#}$ & 0.02 \\
\hline
\end{tabular}

Values are median (range). ${ }^{*},{ }^{* *},{ }^{\#} P=0.01$;

${ }^{\dagger} P=0.006$

* $P=0.002$ between treatment groups

layer, reduced safranin $\mathrm{O}$ staining was seen, and occasionally, empty chondrocyte lacunae were present in local slightly fibrillated areas.

In the 6 months implant group, 24 months implant group, and meniscectomy group, tibial cartilage degeneration was more severe, more diffuse, and more variable compared to femoral cartilage degeneration. On the femoral side, the degenerated areas were more localized and similar (less variable) in all specimens. In the peripheral zone of the tibial plateau, beneath the implant or the newly formed tissue, and in the corresponding femoral surface, no or only moderate cartilage degradation was found. In contrast, in the central weight-bearing zone of the tibia and in the corresponding articulating surface on the femur, large local fibrillated defects or deep fibrillated defects over the entire surface were seen (Figs. 6a, d, 7a, d). These locations closely matched the ink-stained areas. Severe depletion of GAGs was demonstrated by the lack of safranin $\mathrm{O}$ staining in both tibial and femoral cartilage.

The cartilage of the three groups showed identical degradation patterns. Clefts of variable depth were present. In the meniscectomy group and 6 months implant group, clefts progressed to the radial zone of the cartilage. However, in the 24 months implant group, the clefts progressed even deeper to the calcified zone. In the degenerated, safranin $\mathrm{O}$ de-stained superficial areas, chondrocytes were lacking or their number was strongly decreased. Below these areas, a thin zone of hypertrophic clustered chondrocytes was seen. No swelling of cartilage was observed in both tibial and femoral cartilage.
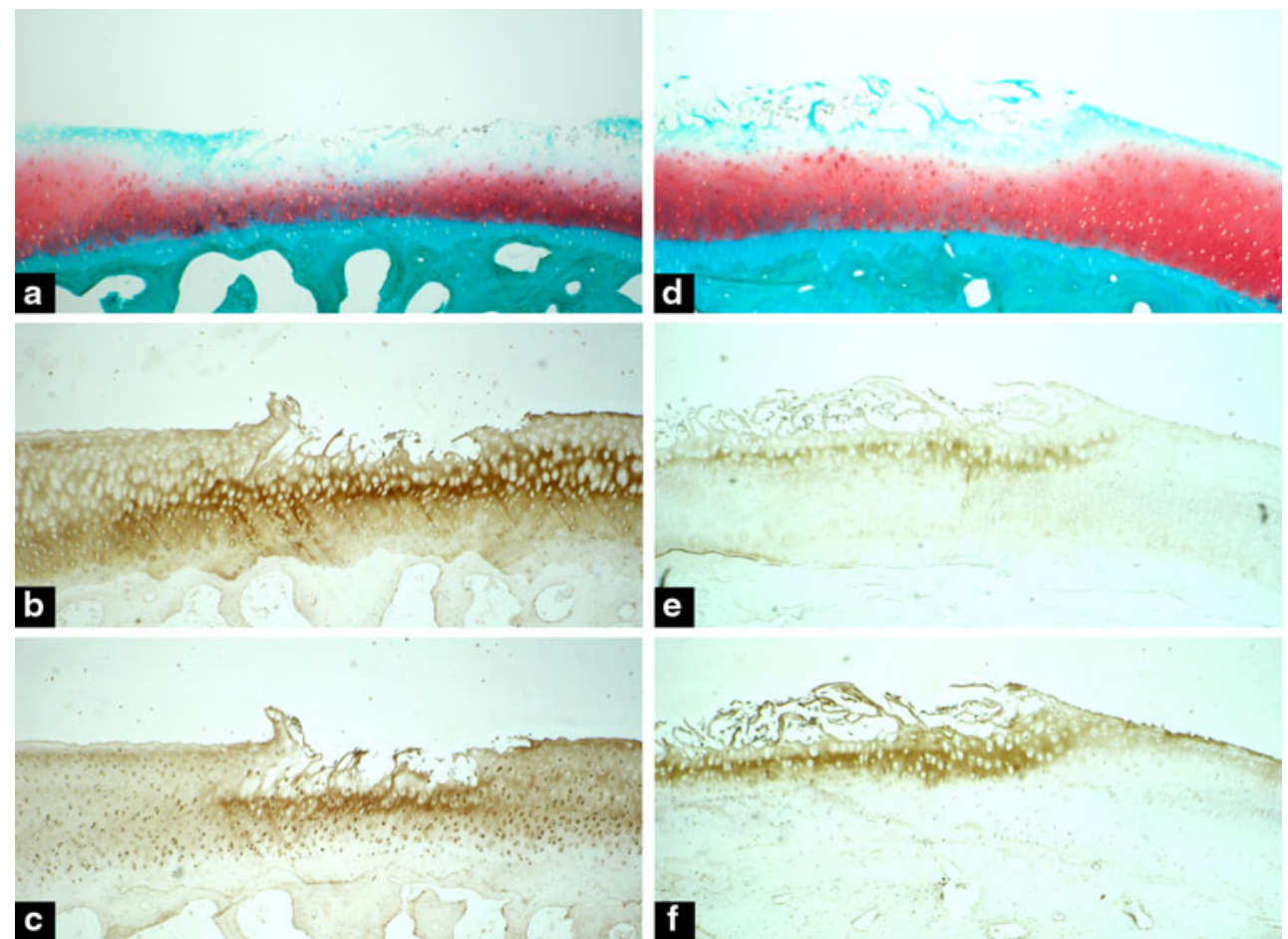

Fig. 6 Adjacent sections of the central weight-bearing zone of the lateral tibial plateaus from the 6 a-c and 24 months $\mathbf{d}-\mathbf{f}$ implant groups. a, d Safranin O-stained sections, b, e Col2-3/4M- and c, f Col2-3/4 $\mathrm{C}_{\text {short }}$-stained sections. Original magnification, $50 \times$ 

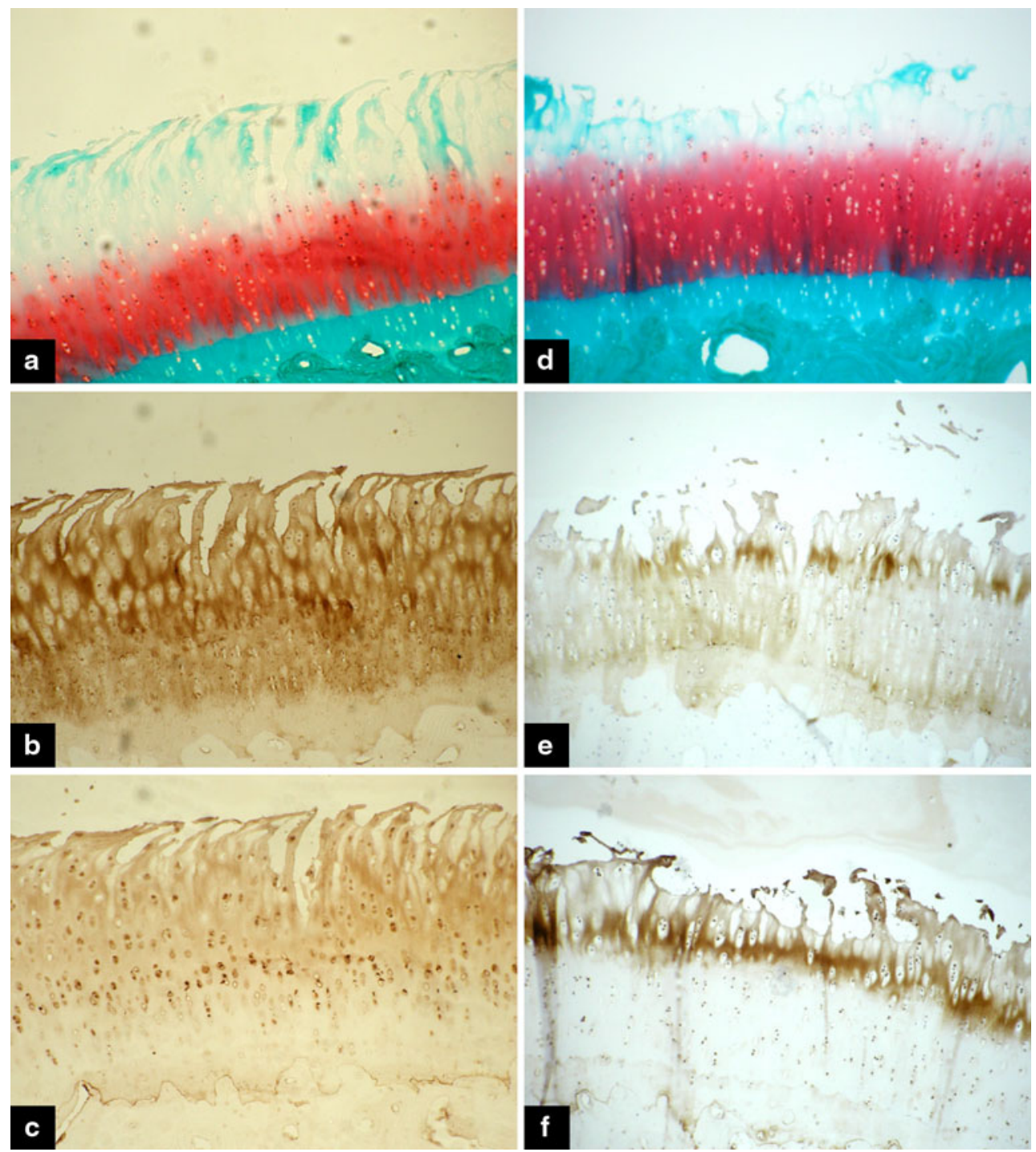

Fig. 7 Adjacent sections from lateral femoral condyles of the meniscectomy a-c and 24 months d-f implant groups. a, d Safranin O-stained sections, b, e Col2-3/4M-,and c, f Col2-3/4 $\mathrm{C}_{\text {short }}$-stained sections. Original magnification, $50 \times$

The 6 months implant group and the meniscectomy group showed comparable cartilage degeneration. However, in the 24 months implant group, cartilage degeneration was more severe compared to the other two groups.

No differences in Mankin scores between the nonoperated medial side and the lateral side were found for both native meniscus group and 6 months implant group. However, in both the 24 months implant group and the meniscectomy group, significantly lower Mankin scores were found for the medial tibia plateau compared to the lateral tibia plateau (Table 2).

\section{Immunohistochemistry}

In the nonoperated medial compartments and in the native meniscus group, Col2- $3 / 4 \mathrm{C}_{\text {short }}$ (cleaved type II collagen) and Col2-3/4M (denatured type II collagen) staining was almost entirely lacking. Irrespective of the group, a thin Col2- $3 / 4 \mathrm{C}_{\text {short }}$-stained strand was seen on the bone-cartilage interface at the location of the tidemark. In some of the specimens, a diffuse Col2-3/4M staining was found throughout the cartilage without any relation with degradation of the cartilage.

In the meniscectomy group and both meniscus implant groups, similar Col2- $3 / 4 \mathrm{C}_{\text {short }}$ and Col2- $3 / 4 \mathrm{M}$ staining patterns were present. In the peripheral zone of the tibial plateau, beneath the implant or the newly formed tissue, and in the corresponding femoral surface, a moderate Col2$3 / 4 \mathrm{C}_{\text {short }}$ and Col2-3/4M staining was visible at the surface in slightly fibrillated areas.

In the central weight-bearing zone of the tibia and the corresponding articulating surface on the femur, intense 
Table 2 Classification of cartilage degeneration using Mankin score

\begin{tabular}{|c|c|c|c|c|c|c|}
\hline \multirow[t]{2}{*}{ Treatment } & \multicolumn{3}{|l|}{ Femur } & \multicolumn{3}{|l|}{ Tibia } \\
\hline & Medial & Lateral & $P$ value & Medial & Lateral & $P$ value \\
\hline Native meniscus & $1(0-2)$ & $0.5(0-6)^{*}$ & 1.0 & $2(1-5)$ & $1.5(1-3)^{\dagger, *}$ & 0.6 \\
\hline 6 months & $2(2-5)$ & $6(3-10)$ & 0.06 & $3.5(2-10)$ & $6.5(2-11)$ & 0.4 \\
\hline 24 months & $2(1-6)$ & $8(7-15)^{*}$ & 0.02 & $2(2-8)$ & $9(4-12)^{\dagger}$ & 0.03 \\
\hline Meniscectomy & $1(0-5)$ & $7(2-11)$ & 0.08 & $1(1-2)$ & $7(4-11)^{*}$ & 0.02 \\
\hline
\end{tabular}

Values are median (range). ${ }^{*},{ }^{\dagger}$, and ${ }^{\star} P=0.001$ between treatment groups

Table 3 Cartilage thickness $(\mu \mathrm{m})$ measurements

\begin{tabular}{|c|c|c|c|c|c|c|}
\hline \multirow[t]{2}{*}{ Treatment } & \multicolumn{3}{|l|}{ Femur } & \multicolumn{3}{|l|}{ Tibia } \\
\hline & Medial & Lateral & $P$ value & Medial & Lateral & $P$ value \\
\hline Native meniscus & $562.3(515.2-773.6)$ & 459.7 (324.9-714.7) & 0.03 & $593.5(522.9-732.2)$ & $530.7(329.6-631.2)$ & 0.2 \\
\hline 6 months & $560.6(517.2-638.2)$ & $345.6(285.6-455.8)$ & 0.03 & $654.2(592.2-697.8)$ & $398.3(243.4-650.3)$ & 0.06 \\
\hline 24 months & $754.6(517.0-857.2)$ & $363.2(35.3-420.8)$ & 0.03 & $701.5(620.2-823.9)$ & $367.4(30.1-529.0)$ & 0.02 \\
\hline Meniscectomy & $551.6(422.5-698.8)$ & $390.6(265.7-491.9)$ & 0.04 & $660.6(557.5-685.5)$ & $407.5(289.3-686.6)$ & 0.06 \\
\hline
\end{tabular}

Values are median (range)

$\mathrm{Col} 2-3 / 4 \mathrm{C}_{\text {short }}$ and $\mathrm{Col} 2-3 / 4 \mathrm{M}$ staining were particularly found in the transition zone between the safranin O-negative surface zone, where chondrocytes were lacking or were totally absent, and the deeper nondegraded zones of the cartilage (Figs. 6b, c, e, f, 7b, c, e, f). This zone corresponds with the zone where hypertrophic clustered chondrocytes were located. Col2-3/4 $\mathrm{C}_{\text {short }}$ staining was directly related to this zone, the $\mathrm{Col} 2-3 / 4 \mathrm{M}$ extended slightly deeper and more laterally into the cartilage. In addition, at the transition between damaged and nondamaged cartilage, as well as under localized cartilage defects, both Col2-3/4 $\mathrm{C}_{\text {short }}$ and $\mathrm{Col} 2-3 / 4 \mathrm{M}$ staining were seen.

In the deeper, nondamaged zones of the cartilage, no or only very slight $\mathrm{Col} 2-3 / 4 \mathrm{C}_{\text {short }}$ and $\mathrm{Col} 2-3 / 4 \mathrm{M}$ labeling was found. These deeper zones were comparable with the native meniscus group.

In both 6 and 24 months meniscus implant groups, the tibial and femoral cartilage showed $\mathrm{Col} 2-3 / 4 \mathrm{C}_{\text {short }}$ and Col2$3 / 4 \mathrm{M}$ staining that progressed deeper into the cartilage compared to the meniscectomy group.

\section{Cartilage thickness}

Within each group, on the femoral side, the cartilage on the lateral condyle was thinner than the cartilage on the medial condyle. However, no difference in cartilage thickness between lateral and medial tibial plateaus was found. Only in the 24 months implant group, cartilage on the medial tibial plateau was thicker than the cartilage on the lateral tibial plateau. A large variation in cartilage thickness between the animals was observed. No differences in cartilage thickness between treatment groups were found (Table 3).

\section{Discussion}

The most important finding of the present study was that after 24 months of implantation of a polymer meniscus, significantly more cartilage damage was found compared with the native, 6 months and meniscectomy group. Degradation of the cartilage matrix (type II collagen network) was the result of both mechanical overloading and localized cell-mediated enzymatic degradation. The longterm tissue differentiation, changes in implant stiffness, and foreign body reactions have been published earlier [41].

In a previous study, we compared articular cartilage damage after meniscectomy and implantation of a porous polymer was compared after 3 and 6 months [38]. It was then speculated that the improved surface properties of the implant over time could have a beneficial effect on chondroprotection. However, this was not found in the present study.

The ink staining, mainly located on the central weightbearing zone of the tibia and the corresponding articulating surface on the femur, strongly suggests that the cartilage damage is the result of abnormal stresses between femur and tibia. Apparently, neither the regenerated meniscus-like tissue that had been formed after meniscectomy nor the polymer implants could prevent this. This would indicate that the initiation of damage is merely a result of overloading of tibia on femur rather than of 
abrasive properties of the foam. If this is the case, then it should be concluded that the initial mechanical characteristics of the implant itself were not sufficient to protect the articular cartilage from overloading. Initially, the compression moduli of the scaffolds are indeed rather low, which might lead to almost similar contact stresses between tibia and femur when compared to meniscectomy [8, 38]. This might explain why no significant differences in Mankin scores between meniscectomy and implant group were observed at short follow-up periods. Although after 24 months, the compression moduli of the implants did not differ from the compression moduli after 6 months [41] and were intermediate between the scaffold before implantation and native meniscus tissue, the articular cartilage further deteriorated between 6 and 24 months as observed by ink-stained surface area and Mankin scores.

In addition, the cartilage under the meniscus implant on the tibial plateau was also damaged. Even after 24 months, some parts of the implant were not covered with tissue and by that might have introduced friction between implant and cartilage and might explain the damage outside the central weight-bearing zone.

The localization of both (cleaved and denatured collagen) epitopes strongly suggests that a more general disruption of the matrix under the influence of mechanical overloading occurs while simultaneously localized cellmediated enzymatic degradation occurs. Both cleaved and denatured collagen increase as the severity of OA increases [18]. It has been shown that chondrocyte death is also associated with cartilage matrix degradation [33].

There are only a few studies dealing with histological cartilage evaluation after meniscal replacement with a similar long-term follow-up. Most studies, even with shortor medium-term follow-up, did not even find a significant difference in comparison with meniscectomized knees $[11,13,22,23,34,44]$. To our knowledge, so far there is only one study that shows a chondroprotective effect after meniscal replacement. Kobayashi et al. [14] reported on the use of hydrogel menisci in a small animal model. Using both gross and histological parameter, they compared lateral meniscal transplantation using a hydrogel implant versus lateral meniscectomy in 19 rabbits with a 2-year follow-up. They found slight macroscopic and microscopic changes in the articular cartilage in the early stages (4 and 6 months). However, the meniscal hydrogel group showed little progression of cartilage degeneration, by both gross and histological examinations, at 1- and 2-year follow-up. Conversely, the meniscectomy group exhibited severe cartilage wear and osteophyte formation at 1 year. The hydrogel implants demonstrated good wear characteristics and no evidence of infection or immune-mediated response.
The small size of the Beagle knee joints made the surgery difficult. The actual goal of restoring the conditions of the normal knee with a meniscal implant has been shown to be difficult to achieve. Precise positioning of the anterior and posterior horn seems to be mandatory for achieving an adequate pressure distribution by the meniscal implant. Placement of the posterior horn in a nonanatomic location has been shown to cause a significant increase in the normal pressure over all flexion angles [29], leading to degenerative cartilage changes that are even worse than that after meniscectomy [40].

Besides the problem of malpositioning, a correct size of the implant has been frequently mentioned to be essential for a successful meniscal transplantation. In our previous studies, the implants were cut and modeled by hand from a porous polymer block to the shape of the native meniscus during surgery [38, 39]. Aiming to reduce variation, in the present study, polymer implants have been produced using a mold, providing standard-shaped, identical-sized, and smooth-surfaced scaffolds. However, an undersized implant may be trapped beneath the femoral condyle and thereby subjected to disproportionally high forces, predisposing the implant to failure. Conversely, an oversized implant may have a loose fit around the femoral condyle, and therefore mechanically ineffective [21]. Only a mismatch of less than $10 \%$ of the original meniscus seems to be acceptable [6].

Most animal studies report a high incidence of implant lesions at longer follow-up. Recently, it has been shown that these lesions appear to be related to the implant fixation technique used [17]. Using two different fixation techniques allowing comparison between a rigid, transtibial horn fixation and a less rigid, more physiological fixation to the meniscal ligaments, a higher incidence of tears and implant dislocations and worse implant and joint appearances were observed in cases where transtibial fixation of the horns was used. It is likely that a too rigid horn fixation does not allow for physiological movement of the meniscal implant and resulted in high mechanical stresses on the implant, leading to a higher incidence of implant failure and cartilage degradation.

\section{Conclusions}

The long-term effects of implantation of a biodegradable polymer meniscus implant on articular cartilage degeneration were evaluated and compared to articular cartilage degeneration after meniscectomy. After 24 months, significantly more cartilage damage was found compared with the native, 6 months and meniscectomy group. Degradation of the cartilage matrix (type II collagen network) was the result of both mechanical overloading as well as localized cell-mediated enzymatic degradation. The severity 
of degeneration was highly variable between animals. Further improvements in the implant, model and surgical technique are essential for adequate protection of the articular cartilage. Clinical application of a porous polymer implant for total meniscus replacement is not supported by this study.

Acknowledgments The authors wish to thank Léon Driessen for technical assistance and René van der Venne for developing the software for the cartilage thickness measurements. This work was financially supported by the Dutch Arthritis Association (Reumafonds).

Conflict of interest The authors declare that they have no conflict of interest.

Open Access This article is distributed under the terms of the Creative Commons Attribution Noncommercial License which permits any noncommercial use, distribution, and reproduction in any medium, provided the original author(s) and source are credited.

\section{References}

1. Aagaard H, Jorgensen U, Bojsen MF (1999) Reduced degenerative articular cartilage changes after meniscal allograft transplantation in sheep. Knee Surg Sports Traumatol Arthrosc 7:184-191

2. Bae WC, Wong VW, Hwang J, Antonacci JM, NugentDerfus GE, Blewis ME, Temple-Wong MM, Sah RL (2008) Wear-lines and split-lines of human patellar cartilage: relation to tensile biomechanical properties. Osteoarthr Cartil 16:841-845

3. Bruns J, Kahrs J, Kampen J, Behrens P, Plitz W (1998) Autologous perichondral tissue for meniscal replacement. J Bone Joint Surg Br 80:918-923

4. Bruns J, Kampen J, Kahrs J, Plitz W (2000) Autologous meniscus replacement with rib perichondrium Experimental results. Orthopade 29:145-150

5. Crook TB, Ardolino A, Williams LA, Barlow IW (2009) Meniscal allograft transplantation: a review of the current literature. Ann R Coll Surg Engl 91:361-365

6. Dienst M, Greis PE, Ellis BJ, Bachus KN, Burks RT (2007) Effect of lateral meniscal allograft sizing on contact mechanics of the lateral tibial plateau: an experimental study in human cadaveric knee joints. Am J Sports Med 35:34-42

7. Elliott DM III, JR Setton LA, Scully SP, Vail P, Guilak F (2002) Joint degeneration following meniscal allograft transplantation in a canine model: mechanical properties and semiquantitative histology of articular cartilage. Knee Surg Sports Traumatol Arthrosc 10:109-118

8. Heijkants RG, van Calck RV, de Groot JH, Pennings AJ, Schouten AJ, van Tienen TG, Ramrattan N, Buma P, Veth RP (2004) Design, synthesis and properties of a degradable polyurethane scaffold for meniscus regeneration. J Mater Sci Mater Med 15:423-427

9. Heijkants RG, van Calck RV, van Tienen TG, de Groot JH, Buma P, Pennings AJ, Veth RP, Schouten AJ (2005) Uncatalyzed synthesis, thermal and mechanical properties of polyurethanes based on poly (epsilon-caprolactone) and 1,4-butane diisocyanate with uniform hard segment. Biomaterials 26:4219-4228

10. Kang SW, Son SM, Lee JS, Lee ES, Lee KY, Park SG, Park JH, Kim BS (2006) Regeneration of whole meniscus using meniscal cells and polymer scaffolds in a rabbit total meniscectomy model. J Biomed Mater Res A 77:659-671
11. Kelly BT, Robertson W, Potter HG, Deng XH, Turner AS, Lyman S, Warren RF, Rodeo SA (2007) Hydrogel meniscal replacement in the sheep knee: preliminary evaluation of chondroprotective effects. Am J Sports Med 35:43-52

12. Khetia EA, McKeon BP (2007) Meniscal allografts: biomechanics and techniques. Sports Med Arthrosc 15:114-120

13. Klompmaker J, Veth RP, Jansen HW, Nielsen HK, de Groot JH, Pennings AJ (1996) Meniscal replacement using a porous polymer prosthesis: a preliminary study in the dog. Biomaterials 17:1169-1175

14. Kobayashi M, Chang YS, Oka M (2005) A two year in vivo study of polyvinyl alcohol-hydrogel (PVA-H) artificial meniscus. Biomaterials 26:3243-3248

15. Kohn D, Rudert M, Wirth CJ, Plitz W, Reiss G, Maschek H (1997) Medial meniscus replacement by a fat pad autograft. An experimental study in sheep. Int Orthop 21:232-238

16. Kohn D, Wirth CJ, Reiss G, Plitz W, Maschek H, Erhardt W, Wulker N (1992) Medial meniscus replacement by a tendon autograft.Experiments in sheep. J Bone Joint Surg Br 74:910-917

17. Kon E, Chiari C, Marcacci M, Delcogliano M, Salter DM, Martin I, Ambrosio L, Fini M, Tschon M, Tognana E, Plasenzotti R, Nehrer S (2008) Tissue engineering for total meniscal substitution: animal study in sheep model. Tissue Eng Part A 14:1067-1080

18. Konttinen YT, Ma J, Ruuttilal P, Hukkanen M, Santavirta S (2005) Chondrocyte-mediated collagenolysis correlates with cartilage destruction grades in osteoarthritis. Clin Exp Rheumatol 23:19-26

19. Mankin HJ, Dorfman H, Lippiello L, Zarins A (1971) Biochemical and metabolic abnormalities in articular cartilage from osteo-arthritic human hips II. Correlation of morphology with biochemical and metabolic data. J Bone Joint Surg Am 53:523-537

20. McDermott ID, Amis AA (2006) The consequences of meniscectomy. J Bone Joint Surg Br 88:1549-1556

21. McDermott ID, Sharifi F, Bull AM, Gupte CM, Thomas RW, Amis AA (2004) An anatomical study of meniscal allograft sizing. Knee Surg Sports Traumatol Arthrosc 12:130-135

22. Messner K (1994) Meniscal substitution with a teflon-periosteal composite graft: a rabbit experiment. Biomaterials 15:223-230

23. Messner K (1994) The concept of a permanent synthetic meniscus prosthesis: a critical discussion after 5 years of experimental investigations using dacron and teflon implants. Biomaterials $15: 243-250$

24. Mora G, Alvarez E, Ripalda P, Forriol F (2003) Articular cartilage degeneration after frozen meniscus and Achilles tendon allograft transplantation: experimental study in sheep. Arthroscopy 19:833-841

25. Noyes FR, Barber-Westin SD (2005) Meniscus transplantation: indications, techniques, clinical outcomes. Instr Course Lect 54:341-353

26. Pastoureau P, Chomel A (2004) Methods for cartilage and subchondral bone histomorphometry. Methods Mol Med 101:79-91

27. Rath E, Richmond JC, Yassir W, Albright JD, Gundogan F (2001) Meniscal allograft transplantation. Two-to eight-year results. Am J Sports Med 29:410-414

28. Rijk PC, Tigchelaar-Gutter W, Bernoski FP, Van Noorden CJ (2004) Histologic changes in articular cartilage after medial meniscus replacement in rabbits. Arthroscopy 20:911-917

29. Sekaran SV, Hull ML, Howell SM (2002) Nonanatomic location of the posterior horn of a medial meniscal autograft implanted in a cadaveric knee adversely affects the pressure distribution on the tibial plateau. Am J Sports Med 30:74-82

30. Sohn DH, Toth AP (2008) Meniscus transplantation: current concepts. J Knee Surg 21:163-172

31. Stollsteimer GT, Shelton WR, Dukes A, Bomboy AL (2000) Meniscal allograft transplantation: a 1 to 5-year follow-up of 22 patients. Arthroscopy 16:343-347 
32. Szomor ZL, Martin TE, Bonar F, Murrell GA (2000) The protective effects of meniscal transplantation on cartilage. An experimental study in sheep. J Bone Joint Surg Am 82:80-88

33. Thomas CM, Fuller CJ, Whittles CE, Sharif M (2007) Chondrocyte death by apoptosis is associated with cartilage matrix degradation. Osteoarthr Cartil 15:27-34

34. Toyonaga T, Uezaki N, Chikama H (1983) Substitute meniscus of Teflon-net for the knee joint of dogs. Clin Orthop 291-297

35. van Arkel ER, de Boer HH (2002) Survival analysis of human meniscal transplantations. J Bone Joint Surg Br 84:227-231

36. van Tienen TG, Hannink G, Buma P (2009) Meniscus replacement using synthetic materials. Clin Sports Med 28:143-156

37. van Tienen TG, Heijkants RG, de Groot JH, Pennings AJ, Poole AR, Veth RP, Buma P (2003) Presence and mechanism of knee articular cartilage degeneration after meniscal reconstruction in dogs. Osteoarthr Cartil 11:78-84

38. van Tienen TG, Heijkants RG, de Groot JH, Pennings AJ, Schouten AJ, Veth RP, Buma P (2006) Replacement of the knee meniscus by a porous polymer implant: a study in dogs. Am J Sports Med 34:64-71

39. van Tienen TG, Heijkants RG, de Groot JH, Schouten AJ, Pennings AJ, Veth RP, Buma P (2006) Meniscal replacement in dogs. Tissue regeneration in two different materials with similar properties. J Biomed Mater Res B Appl Biomater 76:389-396
40. von Lewinski G, Kohn D, Wirth CJ, Lazovic D (2008) The influence of nonanatomical insertion and incongruence of meniscal transplants on the articular cartilage in an ovine model. Am J Sports Med 36:841-850

41. Welsing RT, van Tienen TG, Ramrattan N, Heijkants R, Schouten AJ, Veth RP, Buma P (2008) Effect on tissue differentiation and articular cartilage degradation of a polymer meniscus implant: a 2-year follow-up study in dogs. Am J Sports Med 36:1978-1989

42. Wilson $\mathrm{W}$, van Burken $\mathrm{C}$, van Donkelaar C, Buma P, van Rietbergen B, Huiskes R (2006) Causes of mechanically induced collagen damage in articular cartilage. J Orthop Res 24:220-228

43. Wirth CJ, Peters G, Milachowski KA, Weismeier KG, Kohn D (2002) Long-term results of meniscal allograft transplantation. Am J Sports Med 30:174-181

44. Wood DJ, Minns RJ, Strover A (1990) Replacement of the rabbit medial meniscus with a polyester-carbon fibre bioprosthesis. Biomaterials 11:13-16

45. Zaffagnini S, Giordano G, Vascellari A, Bruni D, Neri MP, Iacono F, Kon E, Presti ML, Marcacci M (2007) Arthroscopic collagen meniscus implant results at 6 to 8 years follow up. Knee Surg Sports Traumatol Arthrosc 15:175-183 